\title{
RFI SURVEY FOR THE GIANT METREWAVE RADIO TELESCOPE IN INDIA
}

\section{G. SWARUP, AND}

T.L. VENKATASUBRAMANI

Tata Institute of Fundamental Research

TIFR Centre, P.O. Box 1234, I.I.Sc. Campus, Bangalore 560 012, India.

\begin{abstract}
A Giant Meterwave Radio Telescope (GMRT) is being set up at Khodad about $80 \mathrm{~km}$ north of Pune in India for operation in the frequency range of about 30 to $1500 \mathrm{MHz}$. It is to be completed by 1992 and is being designed to investigate many outstanding problems in the fields of galactic and extragalactic astronomy. We present here measurements of man-made radio frequency interference (RFI) conducted at the GMRT site in 1985 and 1988. It is seen that highly sensitive radio astronomy observations can still be made at selected bands in the above frequency range because of the relatively low level of RFI in India. However, this advantage may not remain for more than a decade or two.
\end{abstract}

\section{INTRODUCTION}

The GMRT will consist of 34 fully steerable parabolic dishes of $45 \mathrm{~m}$ diameter each. It will form an earth-rotation synthesis interferometer of about $25 \mathrm{~km}$ in extent. It will be operating in the frequency bands of 37.5-38.25, 152$154,230-235,322-328.6,608-614$ and $1400-1427 \mathrm{MHz}$. All the bands (except 230$235 \mathrm{MHz}$ ) are internationally identified bands for radio astronomy observations and are being coordinated locally for GMRT usage. However, for certain astronomical investigations, it is important to use a much wider bandwidth than can be coordinated against RFI. Hence, it is desirable to build a receiver system with a large dynamic range and sufficient frequency resolution. It is therefore planned to connect the $16 \mathrm{MHz}$ baseband output of each antenna to a 512 channel FFT processor for each of the two orthogonal polrizations. The signals from all the 34 antennas will be cross-correlated for all the 512 frequency channels for each of the two polarizations. This spectro- correlator system (FX) is advantageous for spectral-line radio astronomy observations and also for rejection of RF interference expected at metre wavelengths over a $16 \mathrm{MHz}$ bandwidth. A wide band system is also planned in the frequency range of about $1000-1400 \mathrm{MHz}$ to study neutral hydrogen emission from galaxies with high redshift.

The harmful level of interference for radio astrongmy continuum observations is calculated to be about -198 and $-180 \mathrm{db} \mathrm{W} / \mathrm{m}^{2}$ at 38 and 1413 $\mathrm{MHz}$ respectively for $2000 \mathrm{~s}$ integration (CCIR Report 224- 6, 1986), and the 
levels are about $20 \mathrm{db}$ lower for spectral line observations. A synthesis array, such as the GMRT, can tolerate about 10 to $30 \mathrm{db}$ higher interference levels depending on the antenna spacings (Thompson, 1982). As an example, for a multi-channel receiver with dynamic range of about $50 \mathrm{db}$, it is required that the level of the strongest interfering signals be lower than about $(-194+20+50)=$ $-124 \mathrm{db} \mathrm{W} / \mathrm{m}^{2}$ at $150 \mathrm{MHz}$. This RFI level may however lead to intermodulation noise as it is equal to the receiver noise for a system temperature of about $300 \mathrm{~K}$, a bandwidth of $10 \mathrm{MHz}$ and a sidelobes gain of $1 / 3$ at $150 \mathrm{MHz}$. For longer integration it is therefore required that RFI signals near $150 \mathrm{MHz}$ are about 10 or $20 \mathrm{db}$ lower than the above value over the $10 \mathrm{MHz}$ band. For a stronger RFI level, the bandwidth of the receiver may have to be restricted so as to exclude the strong signals.

The instrumentation built for RFI survey at the GMRT site to a sensitivity of about $-150 \mathrm{dbW} / \mathrm{m}^{2}$ is described in Section 2, and the results are presented in Section 3 .

\section{BRIEF DESCRIPTION OF THE SYSTEMS:}

Two types of surveys were made:

(a) A broad-band spectral survey was made using a log-periodic antenna (70 to 200 or 130 to $350 \mathrm{MHz}$ ) feeding a broad-band amplifier (TRW CA3820), whose output was connected to a spectrum analyser (Tektronix 7L14). The noise floor achieved with this set-up was around $-150 \mathrm{dBW} / \mathrm{m}^{2}$, with a resolution bandwidth of $30 \mathrm{kHz}$. In addition dipoles were also used around $40 \mathrm{MHz}$.

(b) A Dicke-switched receiver operating in RF bands at 145-155, 225-235 and $320-330 \mathrm{MHz}$ was used. Any 3.6 MHz RF band was converted into 12 adjacent baseband channels of $300 \mathrm{kHz}$ bandwidth and the outputs of these were recorded in a PC-based data-acquisition system. The sensitivity achieved was around $-160 \mathrm{dBW} / \mathrm{m}^{2}$.

\section{SUMMARY OF RESULTS:}

The results of Broad-band RFI Surveys made in 1985 and 1988 at the GMRT site are given in Figure 1. Crosses and circles show the peak power density of various signals as measured over a period of about one week in 1985 and 1988, respectively. For the 1988 data we show values of RFI only at the frequencies not seen in 1985 . Note that the measurements of 1985 covered 40 to $200 \mathrm{MHz}$.

For comparison, Figure 2 shows the measurements taken with the same system in 1988 about $10 \mathrm{kms}$ from Pune, which is $80 \mathrm{~km}$ south of the GMRT site and is likely to be the main source of RFI.

Additionally, data were taken at the GMRT site for about 1200 hours, spread over 4 months during 1988, using the PC-based receiver system described in Section 2(b). All bands were found to be free of interference to the sensitivity limit of the system, except for 149.7 to $150.2 \mathrm{MHz}$, where RFI was present for about $20 \%$ of the time, reaching a peak level of around $-130 \mathrm{dBW} / \mathrm{m}^{2}$ in the 150.0 $\pm 0.15 \mathrm{MHz}$ band. 


\section{CONCLUSIONS:}

The selected bands for the GMRT at metre wavelengths are relatively free from interference near the GMRT site to the sensitivity limit of about $-150 \mathrm{db}$ $\mathrm{W} / \mathrm{m}^{2}$. More measurements are planned over the frequency range $30-1500 \mathrm{MHz}$ and with improved sensitivity.

\section{REFERENCES}

[1] CCIR, 1986, "Interference Protection Criterion for the Radio Astronomy Service-Report 224-6", Recommendations and Reports of the CCIR, XVIth Plenary Assembly, Dubrovnik, International Telecommunication Union, Geneva, $\mathrm{p} 493$.

2] Thompson, A.R., 1982, IEEE Trans. AP, 30, p450. 

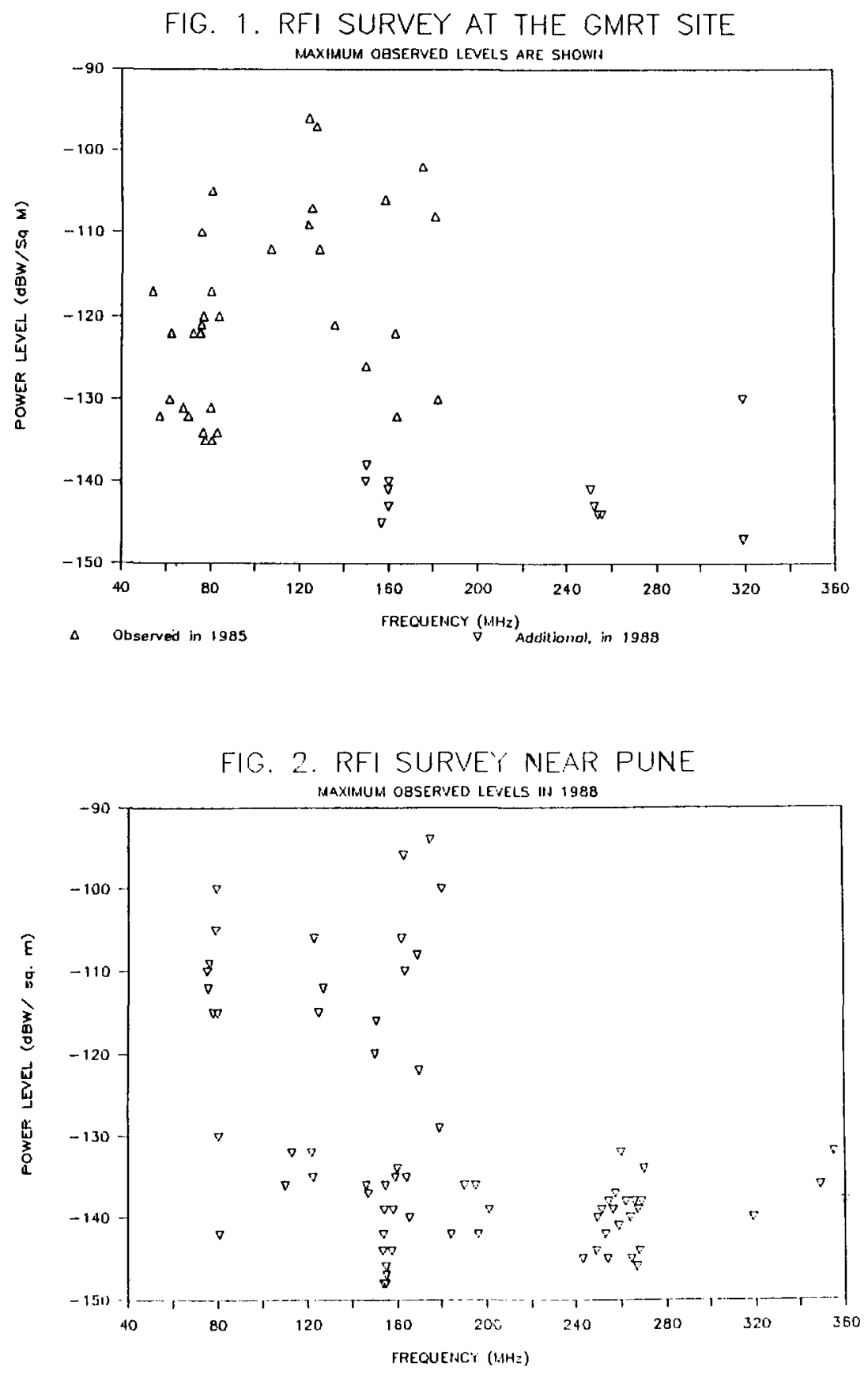